\title{
Tunable millimeter-wave phase shifter based on dielectric elastomer actuation
}

\author{
P. Romano, ${ }^{1, a)}$ O. Araromi, ${ }^{2}$ S. Rosset,${ }^{2}$ H. Shea ${ }^{2}$ and J. Perruisseau-Carrier ${ }^{1, a)}$ \\ ${ }^{1}$ Adaptive MicroNano Wave Systems, École Polytechnique Fédérale de Lausanne (EPFL), 1015 Lausanne, \\ Switzerland \\ ${ }^{2}$ Microsystems For Space Technologies Laboratory, École Polytechnique Fédérale de Lausanne (EPFL), \\ 2002 Neuchâtel, Switzerland
}

(Received 6 November 2013; accepted 3 January 2014; published online 15 January 2014)

\begin{abstract}
A very low-loss tunable millimeter-wave phase shifter driven by dielectric elastomer actuators (DEAs) is presented. The device consists of a fixed coplanar waveguide (CPW) and two metallic loading strips suspended on an elastomer membrane. The horizontal offset between the CPW and the strips is dynamically controlled by integrated DEAs. The variable interaction between the CPW and the loading strips results in a change in the effective permittivity, thereby providing analogcontrolled true-time-delay. The design, fabrication, and measurements of this phase shifter based on DEAs are presented, demonstrating state-of-the-art phase shift to loss performance, achieving 235\%/dB at $35 \mathrm{GHz}$. (C) 2014 AIP Publishing LLC. [http://dx.doi.org/10.1063/1.4862272]
\end{abstract}

Reconfigurable phase shifters are key components in phased array antennas for microwave (MW) and millimeterwave (MMW) communications, radars, and remote sensing systems. Dynamic reconfiguration of MW and MMW devices is in fact becoming a prime need, notably for updating in real time antenna characteristics (e.g., coverage, polarization, frequency of operation), as needed both in earth terminals and satellite antennas. ${ }^{1,2}$

In this context, low-loss reconfigurability, complexity, and cost are driving factors in the choice of a given technology. Reconfigurable phase shifters are currently implemented using several technologies, including monolithic microwave integrated circuit (MMIC), ferromagnetic, and RF-MEMS. $^{2}$ All these technologies present peculiar advantages and disadvantages, which can often result in increased cost, loss, and complexity when high-performance reconfiguration capabilities are needed at MW and MMW. MMIC performs well in terms of reliability, switching speed, and power handling, but the main drawback of this technology is related to the high RF losses $(>5 \mathrm{~dB})$. Ferrite phase shifters are suitable to high power applications and can achieve high performance in terms of reliability and radiation tolerance; however, they are bulky, expensive, and have significant DC power consumption. Metal MEMS-based phase shifters exhibit very low insertion loss, high linearity, and low power consumption, but their use is still limited by their reliability issues and low switching speed. They also rely on advanced cleanroom fabrication processes.

Here, we propose the use of dielectric elastomer actuators (DEAs) to implement mechanical reconfiguration. DEAs are stretchable actuators capable of very large strains (over 200\%). ${ }^{3}$ They consist of thin elastomer membranes sandwiched between two compliant electrodes. ${ }^{4,5}$ Applying a voltage bias across the electrodes results in thickness compression and in-plane expansion of the membrane as a result

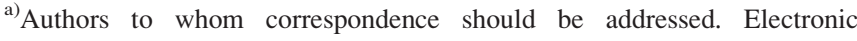
addresses: pietro.romano@epfl.ch and julien.perruisseau-carrier@epfl.ch
}

of electrostatic interactions and the material incompressibility. In our design, we utilize the in-plane expansion to displace loading elements in the electromagnetic (EM) active area, thus achieving a phase shifter reconfiguration. Such an approach allows for isolation of the actuator parts from the EM-active area, resulting in reconfigurable devices with losses comparable to their fixed counterparts. DEAs possess appealing properties for mechanical reconfiguration, ${ }^{3-5}$ including inexpensive materials and fabrication, high power densities, relatively large (analog) strain outputs, and very low DC power consumption. DEAs currently typically require a voltage in the kilovolt range to achieve large strain actuation. This high voltage is considered an acceptable drawback given the advantages brought by the DEA technology, including their extremely low DC power consumption. The required actuation voltages can be readily obtained using commercial DC-DC converters, with volumes of less than $2 \mathrm{~cm}^{3}$. Moreover, these voltages can be significantly reduced by the use of ultra-thin elastomer films (e.g., $<5 \mu \mathrm{m}$ thick). For instance, replacing a $40 \mu \mathrm{m}$ thick membrane by a stack of ten $4 \mu \mathrm{m}$ thick membranes decreases the actuation voltage by a factor of ten with no reduction in device performance. Lower driving voltages would greatly reduce the cost of the control electronics.

The use of DEAs has seldom been investigated thus far for the development of RF concepts, and the presented device is the only available reconfigurable phase shifter based on this technology. Only basic antenna concepts using DEA reconfiguration have been demonstrated at L-band. ${ }^{6,7}$ Nevertheless, these works do not exploit the analogue, very low-loss and large tuning range offered by DEAs, which can be very favorable at high frequency where other technologies can result in increased losses and complexity. In addition, a soft elastomeric membrane has been used as a flexible substrate in a reconfigurable phase shifter. ${ }^{8}$ However, the elastomeric membrane is passive (i.e., does not provide any type of actuation), and actuation must be provided by external and off-the-shelf, electromagnetic actuators. 


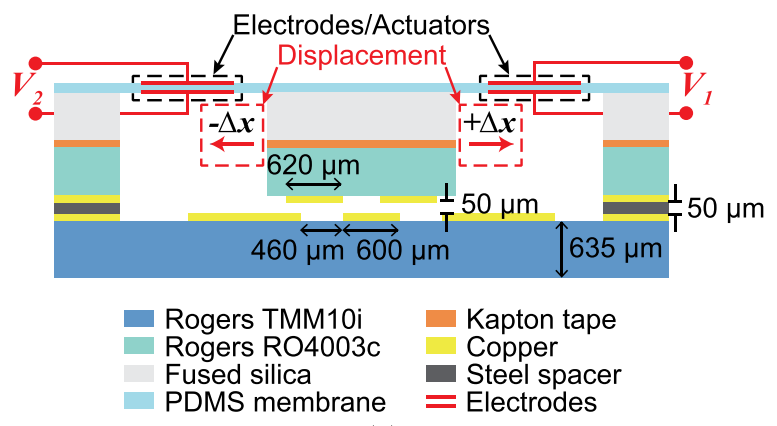

(a)

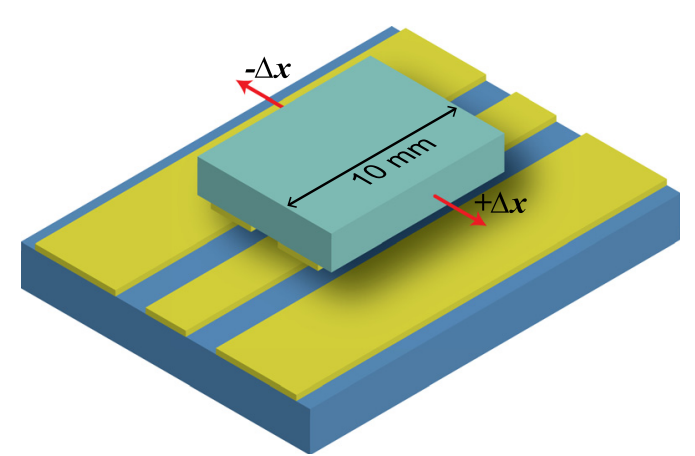

(b)

FIG. 1. Schematic drawing of the DEA-based phase shifter. (a) Crosssection view showing the device assembly and the materials used for the fabrication of the prototype. The air gap between the CPW and the loading strips is obtained using a steel spacer (same thickness as the needed air gap). (b) Simplified 3D view of the CPW and suspended loading part. The maximum differential phase shift is directly proportional to the length of the loading metal strips (here $10 \mathrm{~mm}$ for $180^{\circ}$ phase shift) due to the TTD principle.

The device proposed here is depicted in Fig. 1. It consists of a $50 \Omega$ coplanar waveguide (CPW) loaded by two suspended metallic strips which are supported and horizontally displaced in the $\pm \Delta x$ direction by DEA actuators integrated in the polydimethylsiloxane (PDMS) membrane. The membrane is prestretched equi-biaxially by approximately 1.4 times and fixed to a rigid frame. Compliant electrodes are then placed on both sides of the PDMS membrane (excluding the EM-active area) forming two agonist planar DEAs linked by a central passive (i.e., no electrodes) PDMS membrane (Fig. 1(a)). The compliant electrodes are composed of carbon black particles in a PDMS matrix and are applied using a stamping method. A voltage $V_{1}$ induces a movement $-\Delta x$, while a voltage $V_{2}$ generates a movement of $+\Delta x$ in the opposite direction.

The presented concept implements a quasi-transverse electromagnetic (TEM) transmission line (TL) whose propagation constant $\beta$ can be modified by changing its effective permittivity $\varepsilon_{r, e f f}$. For a TEM mode, $\varepsilon_{r, e f f}$ is equivalent to a weighted average of the different materials' permittivity in the TL cross section, depending on the field distribution in each of them. This basic principle is exploited in the proposed concept, where the horizontal displacement of the suspended lines over the fixed CPW modifies the EM field distribution inside the dielectric substrate and the air gap between the CPW and the loading lines, as illustrated in Fig. 2. This induces a change in the effective permittivity of the equivalent TL: moving from State $A$ to State $B$ (see Fig. 2(a)), $\varepsilon_{r, \text { eff }}$ reduces (the electric field is more concentrated in the air gap

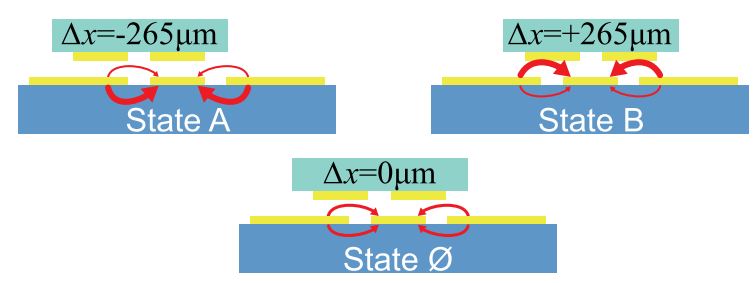

(a)

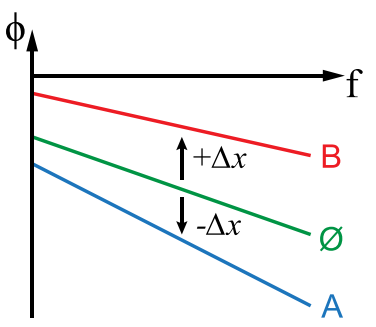

(b)

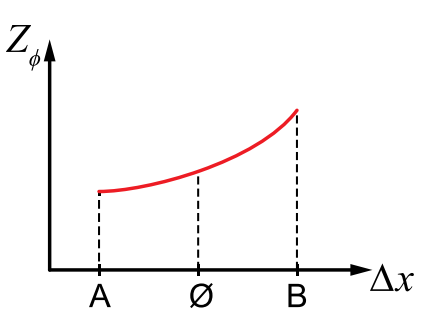

(c)
FIG. 2. Simplified drawing of the phase shifter reconfiguration principle. (a) Three possible states of reconfiguration with a symbolic illustration of the electric field distribution (red arrows): thicker arrows means higher field concentration. States A and B correspond to the minimum and maximum phase shift, respectively. State $\varnothing\left(V_{1}=V_{2}=0 \mathrm{~V}\right)$ is the central state between $\mathrm{A}$ and $\mathrm{B}$ and corresponds to the initial assembly position. (b) Qualitative phase curves corresponding to the states shown in (a). The effective permittivity decreases moving from $\mathrm{A}$ to $\mathrm{B}$, increasing the signal phase according to (1). (c) Qualitative illustration of the characteristic impedance change as a function of the displacement $\Delta x$.

than in the substrate), generating a differential phase shift (Fig. 2(b)) given by

$$
\Delta \phi(A \rightarrow B)=-\left(\beta_{B}-\beta_{A}\right) L_{\phi}=-\omega \mu_{0} \varepsilon_{0}\left(\varepsilon_{r, e f f}^{B}-\varepsilon_{r, e f f}^{A}\right) L_{\phi},
$$

where $L_{\phi}$ is the length of the reconfigurable section, which is the area directly affected by reconfiguration (see Fig. 1(b)). It is clear that this effect is magnified by increasing the dielectric permittivity. For the presented device a dielectric permittivity close to 10 is obtained using the commercial laminate Rogers TMM10i (see Fig. 1(a)), which also guarantees very good mechanical and thermal stability.

The dynamic control of the phase via the change of the effective permittivity of the quasi-TEM mode necessarily comes with a simultaneous variation of the TL characteristic impedance, ${ }^{9}$ which will affect the matching of the phase shifter. Here the characteristic impedance $Z_{\phi}(\Delta x)$ of the active section increases moving from state $A$ to state $B$ as qualitatively illustrated in Fig. 2(c), due to the reduction of the effective permittivity. This results in increased mismatch in at least some of the phase shifter states, since the impedance $Z_{C P W}$ of the feeding CPW is fixed (here to $50 \Omega$ ). The use of matching networks between the feeding CPW and the reconfigurable section could be envisioned, but would not be advantageous in this specific concept. In fact, the matching circuit should be reconfigurable and would come with additional space, loss, and device complexities. Therefore, it is preferable to optimize the design in order to minimize the mismatch. In particular, a "minimum mismatch" design approach is applied. Namely, the dimensions of the CPW and of the loading parts in the reconfigurable section are 
optimized to have the same mismatch in the extreme states of the phase shifter (A and B in Fig. 2(a)). The tuning mechanism ensures that all intermediate states will be better matched, since the impedance changes monotonically with the movement of the loading part as symbolically depicted in Fig. 2(c). After simple calculations, it is possible to demonstrate that the optimal solution in terms of characteristic impedance is given by

$$
Z_{o p t}=\sqrt{Z_{\phi}(A) Z_{\phi}(B)}=Z_{C P W}
$$

The design and optimization are carried out using a fullwave simulation approach. The main objective is to optimize the dimensions of the CPW TL and of the reconfigurable loading part in order to maximize the phase shift to loss figure, while simultaneously minimizing the mismatch. In particular, the optimization process is based on the maximization of two figures of merit (FoMs): the maximum differential phase shift per unit length expressed by (3) and the maximum differential phase shift per unit length per mismatch given by (4)

$$
\begin{gathered}
F o M_{1}=\frac{\Delta \phi_{\max }}{L_{\phi}}=\frac{\phi_{B}-\phi_{A}}{L_{\phi}} \\
F o M_{2}=\frac{\Delta \phi_{\max }}{L_{\phi}\left|\Gamma_{A, B}\right|}
\end{gathered}
$$

Note that $\Gamma_{A, B}$ in (4) is the maximum reflection coefficient between the reconfigurable and fixed sections, which is the same in A and B states, according to the minimum mismatch approach mentioned above. Both $F_{o M}$ and $F_{O} M_{2}$ are normalized by the length of the active region, allowing for optimization independent of the length of the device. The length of the reconfigurable section can be subsequently selected according to the phase shift requirement for a given application, these two quantities being directly proportional (since the phase shift is of TTD nature).

The different parts of the design are fabricated independently using commercial materials (see Fig. 1(a)) and standard printed circuit board (PCB) manufacturing techniques. The fabricated pieces are assembled manually, resulting in a very low-cost phase shifter. Moreover, the prototype is thought to be modular, allowing the use of the same fixed PCB (CPW TL) with different reconfigurable parts (for testing purposes). The manufactured device is shown in Fig. 3, where the small $\left(\sim 12 \times 3 \mathrm{~mm}^{2}\right)$ EM-active area is highlighted.

The fabricated prototype shown in Fig. 3 is characterized through conventional on-wafer S-parameters measurements using an Agilent E8361A network analyzer and a Cascade Microtech CM2 manual probe station. A thrureflect-line (TRL) calibration is used to remove the effect of the transitions between the coaxial-based network analyzer and the CPW-based device and to place the S-parameters reference planes at the edges of the reconfigurable section. Different actuation states are characterized: no applied voltage $\quad\left(V_{1}=V_{2}=0 \mathrm{~V}\right), \quad V_{1}=1.1,1.2, \ldots, 1.5 \mathrm{kV} \quad$ and $V_{2}=1.1,1.2, \ldots, 1.5 \mathrm{kV}$. It is worth observing that the initial position $\left(V_{1}=V_{2}=0 \mathrm{~V}\right)$ corresponds to the 'State $\varnothing$ ' of

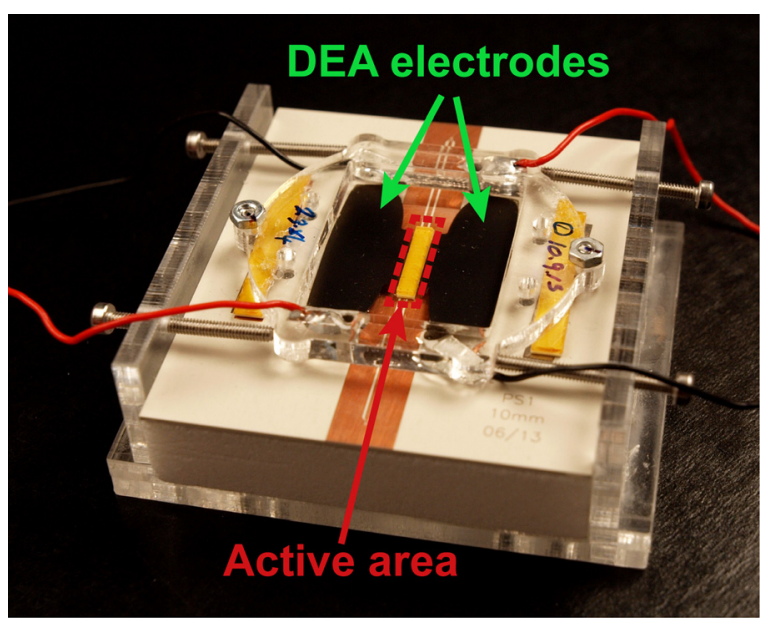

FIG. 3. Picture of the fabricated DEA-based phase shifter. The EM-active area is highlighted with a red dashed-line rectangle, the dimensions are $12 \mathrm{~mm} \times 3 \mathrm{~mm}$. Two DEA segments straddle the EM-active area providing the in-plane actuation. The DEA segments have separate electrical input allowing for independent actuation. The four screws on the perimeter of the device help maintain alignment.

Fig. 2(a), namely, the central position $(\Delta x=0 \mu \mathrm{m})$ between the minimum (state A) and maximum (state B) phase shift. However, due to the manual assembly, some initial displacement can be present in the manufactured prototype, and is characterized comparing measured and simulated curves.

The measured scattering parameters in the range 25-35 GHz are shown in Fig. 4 (note $V_{1}$ voltages are shown as negative and $V_{2}$ voltages shown as positive). Measured curves highlight very good performance in terms of insertion loss $(I L)$, return loss $(R L)$, and maximum differential phase shift $\left(\Delta \phi_{\max }\right)$. The $I L$ is always lower than $1.6 \mathrm{~dB}$ with an average value of $0.83 \mathrm{~dB}$ over the total frequency range. The $R L$ is always better than $11 \mathrm{~dB}$, which means good matching for all the phase shifting states in the whole $10 \mathrm{GHz}$ bandwidth. A maximum differential phase shift $\Delta \phi_{\max } \approx 180^{\circ}$ is achieved at $30 \mathrm{GHz}$, which can be increased at will by proportionally increasing the length of the active region (see. Figs. 1(b) and 3) due to the TTD working principle.

These results demonstrate state-of-the art performance in terms of the most important figure of merit for true-time delay phase shifters, namely, the phase-shift/loss ratio. Indeed, a mean value of $\sim 235^{\circ} / \mathrm{dB}$ is achieved at $35 \mathrm{GHz}$, considerably outperforming MMIC phase shifters at similar frequencies (e.g., $\sim 57^{\circ} / \mathrm{dB}$ at $28 \mathrm{GHz}$ for a 5-bit MMIC phase shifter using InGaAs PIN diodes ${ }^{10}$ ). Moreover, similar performance as lower-loss state-of-the art MEMS reconfigurable phase shifters are obtained. Hacker et al. ${ }^{11}$ demonstrated an average FoM of $\sim 140^{\circ} / \mathrm{dB}$ at $35 \mathrm{GHz}$ for a 3-bit TTD RF MEMS network, while the 2-bit MEMS phase shifter presented by Hayden and Rebeiz ${ }^{12}$ exhibited an average phase shift to loss of $\sim 180^{\circ} / \mathrm{dB}$ at $37.7 \mathrm{GHz}$. In addition, the device proposed here offers a large analog tuning range, which represents an advantage over the digital behavior of its MEMS and MMIC counterparts. These advantages come at the cost of larger actuation voltage and device size. It is however worth noting that higher integration can be obtained, but was not our main goal for this first proof of concept of DEA-based phase shifter. 


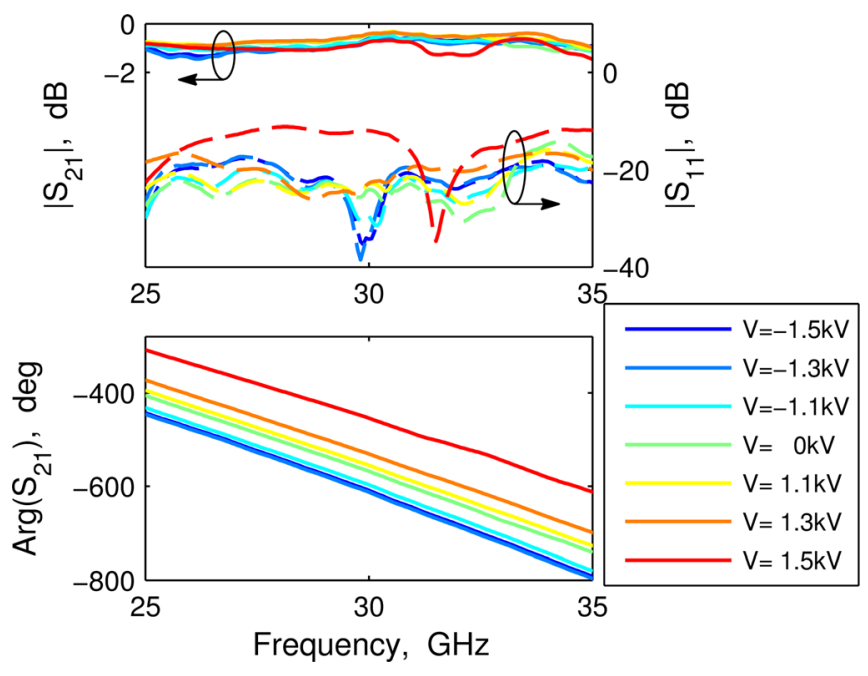

FIG. 4. Measured scattering parameters for different actuation states $\left(V_{1}=V_{2}=0 \mathrm{~V}, V_{1}=1.1,1.3,1.5 \mathrm{kV}\right.$, and $\left.V_{2}=1.1,1.3,1.5 \mathrm{kV}\right)$. The top panel shows the magnitude of S21 (solid lines) and S11 (dashed lines) in $\mathrm{dB}$, highlighting the very low loss and the good matching for all the reconfiguration states, respectively. The bottom panel reports the transmission phase exhibiting the typical TTD behavior. Note $V_{1}$ input voltages shown as negative values and $V_{2}$ as positive in the legend.

Measured scattering parameters are now compared with full-wave simulations from the commercial software Ansys HFSS. Figure 5 shows the simulated result (solid blue line) as a function of the horizontal displacement $\Delta x$, and the measured phase as a function of input voltage (red asterisks) at the central frequency $f_{0}=30 \mathrm{GHz}$. The $\Delta x$ values corresponding to the measured phase data were determined prior to the device assembly and RF characterization, as the particular measurement setup did not allow for measurement of both quantities simultaneously. A good agreement can be seen between the measured and simulated results for negative input voltages (i.e., the " $V_{1}$ " DEA from Fig. 1(a)), whilst a discrepancy exists for the positive input voltages (i.e., the " $V_{2}$ " DEA from Fig. 1(a)). This suggests that the $V_{2}$ DEA displacement during the RF measurement was different to its

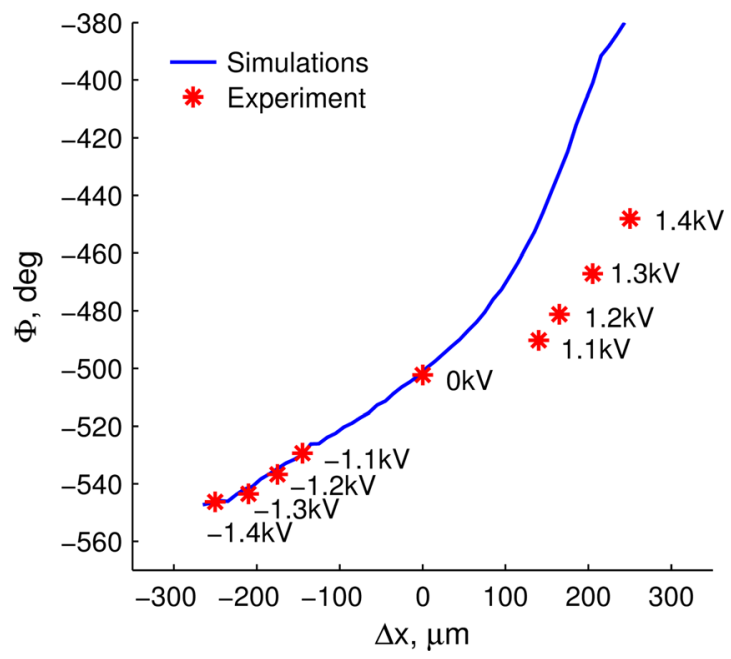

FIG. 5. Comparison between measurements and simulations for the central frequency $f=30 \mathrm{GHz}$ for actuation states $V_{1}=V_{2}=1.1,1.2,1.3,1.4 \mathrm{kV}$ ( $V_{1}$ input voltages shown as negative values). Full-wave simulations are performed using the commercial software HFSS. The $\Delta x$ displacement values for the experiment data were measured prior to the RF measurement.
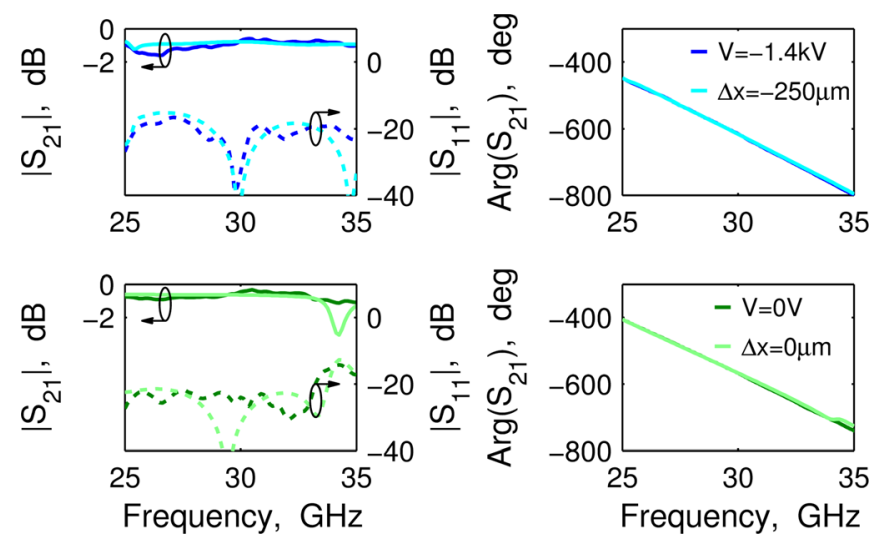

FIG. 6. Comparison between measurements and simulations over the complete frequency range $(25-40 \mathrm{GHz})$ for two different actuation states $\left(V_{1}=1.4 \mathrm{kV}\right.$ and $\left.V_{1}=V_{2}=0 \mathrm{~V}\right)$. The simulated curves are identified by their displacement $\Delta x$.

prior measured value, most likely due to disturbance caused during transport and test preparation. This could be eliminated in future designs by implementing closed-loop control. Figure 6 reports the comparison between measured and simulated scattering parameters over the full frequency range (25-40 GHz) for two of the measured phase shifter states $\left(V_{1}=1.4 \mathrm{kV}\right.$ and $\left.V_{1}=V_{2}=0 \mathrm{~V}\right)$ shown in Fig. 5. The two states shown here correspond to voltages where a very good agreement was achieved in Fig. 5. Nevertheless, in the regions where a discrepancy exists (see Fig. 5), the position of the movable loading part in the simulation must be logically fitted in order to get a good agreement between simulations and measurements.

A TTD reconfigurable phase shifter driven by DEAs has been presented. The phase shifter has been fabricated using commercial materials and standard PCB techniques, resulting in a very low cost prototype. The manufactured device has been characterized using conventional on-wafer measurements and has demonstrated state-of-the-art performance in terms of phase shift per loss compared to other reconfigurable phase shifters. These results demonstrate the potentials of DEAs for very low-loss analogue tuning of electromagnetic devices at millimeter-waves and, possibly, at higher frequencies, where available technologies (e.g., MMIC, MEMS, ferrites, liquid crystals) can come with unacceptable losses. The presented DEA-based mechanical reconfiguration could also be applied to the implementation of other tunable devices in the microwave to terahertz band, such as proposed by Host et al. ${ }^{13}$ and Drysdale et al.. ${ }^{14}$

This research was supported by the European Space Agency (NPI project REACT) and the Swiss National Science Foundation (SNSF) under Grant No. 133583. The authors would like to thank Jean-Christophe Angevain (Antenna and Sub-Millimeter Wave Section, ESA ESTEC) for valuable discussions.

${ }^{1}$ C. Mangenot and G. Toso, European Space Technology Harmonisation Technical Dossier on Array Antennas (European Space Agency, ESTEC, TEC-EEA, Noordwijk, 2012).

${ }^{2}$ J. L. Volakis, Antenna Engineering Handbook, 4th ed. (McGraw-Hill Professional, 2007), p. 21-1. 
${ }^{3}$ I. A. Anderson, T. A. Gisby, T. G. McKay, B. M. O'Brien, and E. P. Calius, J. Appl. Phys. 112, 041101 (2012).

${ }^{4}$ S. Rosset and H. Shea, Appl. Phys. A 110, 281 (2013).

${ }^{5}$ R. Pelrine, R. Kornbluh, Q. Pei, and J. Joseph, Science 287, 836 (2000).

${ }^{6}$ A. Mahanfar, C. Menon, and R. G. Vaughan, Electron. Lett. 44, 1113 (2008).

${ }^{7}$ X.-J. Jiang, S. J. Mazlouman, A. Mahanfar, R. G. Vaughan, and C. Menon, Smart Mater. Struct. 21, 055020 (2012).

${ }^{8}$ S. Hage-Ali, Y. Orlic, N. Tiercelin, R. Sauleau, P. Pernod, V. Preobrazhensky, and P. Coquet, in Proceedings of the IEEE International Microwave Symposium Digest, Montreal, Canada, 17-22 June, 2012, pp. 1-3.
${ }^{9}$ J. Perruisseau-Carrier, R. Fritschi, P. Crespo-Valero, and A. K. Skrivervik, IEEE Trans. Microwave Theory Tech. 54, 383 (2006).

${ }^{10}$ J. G. Yang and K. Yang, IEEE Microwave Wireless Compon. Lett. 21, 151 (2011).

${ }^{11}$ J. Hacker, R. Mihailovich, M. Kim, and J. De Natale, IEEE Trans. Microwave Theory Tech. 51, 305 (2003).

${ }^{12}$ J. S. Hayden and G. M. Rebeiz, IEEE Trans. Microwave Theory Tech. 51, 309 (2003).

${ }^{13}$ N. K. Host, C. Chi-Chih, J. L. Volakis, and F. A. Miranda, IEEE Antennas Propag. Magn. 55, 290 (2013).

${ }^{14}$ T. D. Drysdale, I. S. Gregory, C. Baker, E. H. Linfield, W. R. Tribe, and D. R. Cumming, Appl. Phys. Lett. 85, 5173 (2004). 